\title{
Indicadores de desempenho na consulta
}

Isabel Santos, ${ }^{*}$ Isabel Lourenço Ribeiro**

\section{RESUMO}

As sociedades actuais procuram avaliar, quantificar e classificar a prestação dos serviços com que habitualmente lidam.

Os indicadores de desempenho avaliam elementos mensuráveis da prática clínica diária, permitindo o estabelecimento de prioridades e de decisões políticas de gestão e avaliação das mudanças nos sistemas de saúde. No entanto, são descurados alguns aspectos primordiais como a relação médico-doente, a diferença entre o que o doente percepciona como problema principal e o que o médico é levado a tentar abordar, a comunicação e o contexto social, entre outros.

As autoras pretendem demonstrar os problemas conceptuais e práticos que se colocam na avaliação de desempenho se não se tiver em conta as características fundamentais da praxis do médico de família como a globalidade, a personalização, a coordenação e a integração de cuidados.

Palavras-chave: Indicadores de Qualidade; Indicadores de Desempenho; Medicina Familiar; USF.

\section{INTRODUÇÃO}

A sociedade exige que os serviços públicos demonstrem que o dinheiro investido neles é bem aplicado e, em conformidade, procura avaliar a efectividade das suas prestações. As escolas e as universidades são obrigadas a mostrar resultados, são submetidas a acreditação e têm de fazer relatórios de actividades anualmente. O seu desempenho é objecto de hierarquização. Na era da governação clínica é natural que a qualidade do desempenho dos profissionais seja uma preocupação.

O interesse pelo desempenho dos médicos associa-se aos diferentes movimentos de controlo e garantia de qualidade surgidos da necessidade de prestar contas aos cidadãos e, em consequência, de melhor coordenar os objectivos das instituições. ${ }^{1}$ A política governamental actual, e as reformas levadas a cabo nos Cuidados de Saúde Primários (CSP), indicam que a remuneração deve estar ligada ao desempenho.

O grupo de missão para os cuidados de saúde primários (MCSP) definiu um conjunto de indicadores de desempenho a serem monitorizados nas Unidades de Saúde Familiares (USF) agrupados em diferentes áreas:

* Médica de Família, Chefe de Serviço no CS de Oeiras; Professora Auxiliar Convidada da FCM-UNL

**Médica de Família; Assistente eventual de Clínica Geral.

CS Arcozelo/Boa Nova - Extensão de Saúde de Grijó informação demográfica, disponibilidade, acessibilidade, produtividade, qualidade técnico-científica, efectividade e eficiência. ${ }^{2} \mathrm{O}$ desempenho dos médicos de família, agora nas USF, mais tarde em todo o sistema, estará futuramente sujeito a um maior escrutínio tal como já acontece em diversos países.

Neste contexto, este texto visa descrever os problemas conceptuais e práticos que se colocam numa avaliação de desempenho, mostrar que alguns dos indicadores, agora usados, podendo ser úteis para melhorar alguns componentes do sistema de prestação de cuidados, não servem para avaliar a globalidade do desempenho dos médicos de família, ou melhor, os aspectos que fazem da Medicina Geral e Familiar uma disciplina única e que caracterizam a sua prática: a globalidade, a personalização, a coordenação e a integração de cuidados.

\section{O DESEMPENHO COMO OBJECTO DE AVALIAÇÃO E A SINGULARIDADE DA MEDICINA GERAL E FAMILIAR "General practice is the easiest job in the world to do badly, but the most difficult to do well» Professor Sir Denis Pereira Grey}

O desempenho é o que se faz, o modo de fazer e as circunstâncias que determinam a acção, ou seja, a razão da acção. O desempenho traduz as competências 
adquiridas aplicadas ao mundo real. O que sabemos fazer e o que fazemos é, no entanto, determinado pelos valores da sociedade e pelos valores da profissão. Por isso, a singularidade da MGF advém mais do processo da sua prática clínica do que do seu conteúdo (i.e., como é que a especialidade se concretiza diariamente nas consultas). Sendo uma profissão prática, ${ }^{3}$ a natureza da profissão está continuamente a ser recriada por um processo conjunto entre as pessoas que a praticam - os médicos, e as pessoas em quem se pratica - os pacientes. Os médicos de família, em conjunto com os utentes/pacientes/doentes, criam uma variedade de realidades, em simultâneo, em que as soluções e as decisões clínicas não são simplesmente aplicadas mas mutuamente construídas. Como consequência, «a verdade» ou a realidade clínica não é unilateral, ela é negociada e aberta a múltiplas interpretações.

Assim como um radiologista «vê» mais radiografias do que um clínico e um oftalmologista vê mais retinas do que um internista, também os médicos de família se confrontam, com relativo à vontade, em áreas da fisiopatologia e do comportamento humano pouco claras e indeterminadas em que o tempo é usado como diagnóstico e os aspectos subjectivos têm influência determinante no sofrimento. Cerca de $75 \%$ das pessoas com sintomas não medicamente explicados não têm um diagnóstico no quadro das doenças somatoformes da DSM-IV e, porque os seus sintomas são na sua maioria físicos, eles procuram o médico de família sendo que uma abordagem direccionada para a pesquisa de doença orgânica não lhes resolve o problema e conduz a um maior aumento de consultas e a uma insatisfação crescente. $^{4-6}$

O ouvido interior do MF vai aprendendo a ouvir, a compreender os pensamentos e os sentimentos das pessoas, a interpretar os seus símbolos, as suas palavras e os gestos, os seus movimentos e expressões. Este desempenho requer tempo e reflexão, o indicador de sucesso é expresso numa linguagem singela à saída do consultório «já me sinto muito melhor depois de ter falado consigo» ou «agora já compreendo o que tenho de fazer» ou «já tenho menos dores». Avaliar se a decisão adoptada é a certa quando se adopta este ou aquele estilo, este ou aquele modelo de consulta para aquela pessoa naquela circunstância requer técnicas específicas de recolha de informação, videogravação, entrevistas, audiogravação e não colecções homogéneas de dados. Em Medicina Geral e Familiar só em contextos muito particulares se faz uso de generalizações. Os fluxogramas de decisão não têm em consideração as circunstâncias particulares de cada doente, a multimorbilidade, os nós de ligação dos problemas ou as circunstâncias que alteram o seu equilíbrio dinâmico, cabendo ao médico, em primeira instância, a interpretação do seu significado e, em conformidade, decidir como pode o mapa fornecido pelo doente ajudá-lo a mover-se na vasta extensão do território que este lhe oferece para percorrer.

O desempenho de um médico de família implica um amplo conjunto de competências, direccionadas à gestão de cuidados, à abordagem centrada na pessoa, à tomada de decisão em contexto de baixa incidência e prevalência de doença, à gestão de problemas múltiplos de diferente natureza, quer agudos quer crónicos, à promoção de saúde, à coordenação e integração de cuidados. Sendo uma medicina da pessoa, reconhece que as necessidades individuais têm de se reconciliar com os recursos disponíveis. Os indicadores da consulta, ou melhor das consultas, actividade predominante do médico de família relacionam-se com os 4 domínios (psicológico, social, somático e semiótico) que interferem no equilíbrio dinâmico dos construtos saúde e doença. ${ }^{7}$

Sabemos que o processo de consulta influencia os resultados $^{8,9}$ mas sabemos também que o processo de avaliação, em particular, os indicadores de desempenho, podem modelar o exercício da disciplina. ${ }^{10}$ Por isso os indicadores devem ser definidos, tendo por base as características que fazem da MGF uma especialidade única e que a justificam como base alargada de sistemas de saúde atingindo grande efectividade.

\section{MÉRITOS E DEMÉRITOS DOS INDICADORES DE DESEMPENHO NOS CUIDADOS DE SAÚDE PRIMÁRIOS}

Os indicadores de qualidade são medidas específicas, elementos mensuráveis da prática sobre a qualidade de cuidados, sendo que alguns organismos os diferenciam como indicadores de actividade ou de desempenho ${ }^{11,12}$ capazes de determinar modificações directamente ou indirectamente, a partir de intervenções realizadas na saúde. Os indicadores de desempenho nos CSP poderão levar a estabelecer prioridades e decisões políticas 
de gestão e avaliação das mudanças nas situações de saúde. Isto, se forem gerados de forma regular, dinâmica, praticamente automática e, de preferência, que o utilizador que introduza os dados no sistema o faça de forma intuitiva e sem passos extra que atrasem o desenrolar da consulta.

Os indicadores são habitualmente retirados de revisões casuísticas de registos clínicos ou de fontes de informação sobre rotinas dos serviços relacionadas com a gestão de cuidados. No entanto, é necessário considerar que a maioria dos indicadores são medidas de proximidade («proxy»), facilmente explicadas através da metáfora de condução automóvel. Quando o carro à nossa frente faz sinal de virar à esquerda isso indica uma intenção e não uma acção. Assumir que esse sinal é uma acção pode ter consequências desastrosas para todos os condutores e passageiros em movimento. Por isso é muito importante que um indicador de qualidade diga respeito aos cuidados que dependem do controlo do prestador e das instituições de saúde. ${ }^{13}$ Diversos autores e organismos ${ }^{9-13}$ entendem, pois, não ser aconselhável assumir que o desempenho deste ou daquele médico de família que gere uma vastidão de casos possa ser avaliado apenas, através deste ou daquele indicador de prática. Vários trabalhos, entre os quais se destaca o estudo de Dever, citado por Pineault, mostram a escassa correlação entre recursos destinados ao sistema de saúde e a sua contribuição potencial para a diminuição de mortalidade. ${ }^{14}$

Mas se a construção de indicadores e índices necessita de dados que decorrem dos registos, então é necessário dispor de sistemas de informação adequados que permitam o acesso aos dados e à informação útil. No entanto, apesar de muito se ter avançado nos últimos 3-5 anos em Portugal, ainda muito terá de ser feito de modo a se conseguir obter o que é desejável. Os sistemas de informação deverão ser simples, versáteis e adaptáveis às necessidades reais. No SAM (Sistema de Apoio ao Médico), sistema informático actual mais utilizado, o registo de hemoglobina glicada tem que ser feito na folha de registo dos exames auxiliares de diagnóstico, ou não aparecerá no programa da vigilância da Diabetes. No entanto, na consulta a um doente com Diabetes, o médico, no módulo SOAP, deveria poder, no programa de vigilância de diabetes, escrever este resultado no campo aí existente para esse efeito sem ter que recorrer a outra folha de registo. Esta «disfuncionalidade» também ocorre relativamente ao registo de outros parâmetros como por exemplo o peso ou altura.

Para além das limitações ao uso de indicadores já anteriormente referidas há ainda que considerar os sub registos, os sobre registos e os dados não verdadeiros. Isto é ainda mais verdade quanto a prática clínica é complexa, sendo que na maioria das vezes só se seleccionam indicadores quantitativos facilmente mensuráveis. Mesmo nesta circunstância, é necessário considerar que um indicador pode ter por vezes diferentes leituras. A proporção de doentes diabéticos com tensão arterial inferior a $130 / 80$ pode providenciar informação importante conducente à melhoria de qualidade da prestação de cuidados porque com eles relacionada, mas também pode estar só relacionada com o nível sócio económico da população abrangida ou com a ausência de comorbilidade, pois sabemos que existem diferenças significativas entre médicos e entre sub-regiões de saúde, quer relativamente a este quer a outros aspectos da prática clínica. ${ }^{15}$

Como já foi anteriormente referido, a Medicina Geral e Familiar avalia um indivíduo no seu todo, de uma forma holística, percebendo o papel que aquele mesmo indivíduo desempenha no seio da sua família, da comunidade e do seu local de trabalho. Um médico de família não poderá seguir as Normas de Orientação Clínica de forma rígida sem perceber o doente que tem à sua frente. Será lícito tentar obter um valor de LDL-Colesterol de $70 \mathrm{mg} / \mathrm{dl}$ como alvo nos doentes com $85 \mathrm{e}$ mais anos? ${ }^{16}$ Num idoso de 80 anos, com uma Diabetes Mellitus, com uma insuficiência cardíaca ou uma cardiopatia isquémica, pode-se considerar como aceitável uma HbAlc de $9 \% .{ }^{17}$ Se num ano uma grávida vier à consulta com mais de 14 semanas de gravidez isso pode ser um indicador de mau desempenho do médico ou da sociedade, quando sabemos não existirem problemas de acessibilidade e que o móbil da consulta foi o subsídio da segurança social e não o da vigilância da gravidez? Será legítimo numa sociedade em crise social e económica, num mundo de trabalho impiedoso para as mulheres, pensar que a legislação protectora da maternidade lhes permite cumprir o programa de vigilância no decurso de uma gravidez e fazer todos os exames solicitados, sem colocarem em risco o seu posto de trabalho? Será possível cumprir e fazer cumprir o progra- 
ma de vigilância de gravidez com ecografias nas semanas de gestação adequadas quando os lugares que as efectuam através da Segurança Social, e a uma proximidade razoável dos CS, são cada vez mais escassos e em muitos casos não conseguem dar resposta senão quando marcados com um mês de antecedência?

São algumas destas condicionantes e especificidades na prática Medicina Geral e Familiar que deverão ser tidas em conta para o estabelecimento de critérios de avaliação de qualidade! A ligação destes indicadores de desempenho a incentivos pecuniários e não a formação, sem discussão prévia com os clínicos e consenso quanto à sua selecção, poderá levar o médico a pensar: 1) coloco este doente com valores óptimos de HbAlc de forma a atingir o indicador? 2) não coloco este doente nos valores alvo porque isso irá interferir com a sua qualidade de vida e desrespeita o principio do «primum non nocere»? Isto poderá traduzir-se em dualidades interiores, ocasionando muito maior nível de stress e originar um número muito maior de casos de «burnout».

Sabemos que existe unanimidade quanto ao uso dos indicadores para fins educacionais, para promover a capacidade crítica dos médicos nos Centros de Saúde ou para influenciar políticas sociais que justifiquem a utilidade desta ou daquela locação de recursos. Mas sabemos também que os indicadores, se usados para julgamentos acerca do desempenho de um médico ou com a finalidade de remuneração por mérito, sem reconhecer a influência do contexto nos cuidados, pode desmoralizar os profissionais, de estilo missionário, verdadeiros pilares de qualquer sistema visando a equidade, que estão arduamente a tentar, com recursos limitados, fazer o seu melhor, levando inclusivamente a que zonas mais carenciadas, mais pobres, com mais problemas se vejam a curto prazo desprotegidas de cuidados de saúde primários o que, inevitavelmente, levará a minar a confiança dos cidadãos em tal sistema. . $^{9,10}$

A responsabilidade e o perfil do bom médico, que todos desejam encontrar, não se encontra abrindo a porta da métrica da avaliação. Qualquer sistema, o comportamento dos electrões ou a gestão da multimorbilidade, mostra consideráveis variações. Olhar para os resultados de um grupo e considerar o desempenho de indivíduos ou de organizações como estando abaixo ou acima da média é reducionista e errado, como muito bem expressa a declaração sobre «Indicadores de
Qualidade em Clínica Geral» que o Colégio Inglês de Clínica Geral em $2002^{11}$ sentiu necessidade de fazer. Este breve e sucinto texto esclarece que em qualquer sistema há um conjunto de valores que se encontram para além da amplitude de variação normal do sistema, sendo que todos os indivíduos que se encontram dentro do intervalo definido são avaliados como tendo um desempenho normal ou desejável (e os restantes, ou «outliers», com desempenho acima ou abaixo do normal). Se utilizarmos o critério de três desvios-padrão da média para definir a amplitude do intervalo, reduziremos o risco de classificar erradamente um indivíduo como «outlier», mas aumentaremos o risco de falhar um verdadeiro «outlier». Pelo contrário se estreitarmos a amplitude para um intervalo definido por 2 desviospadrão reduzimos o risco de falhar (de incluir) um verdadeiro «outlier» mas aumentamos o risco de falsamente classificar um indivíduo como tendo um bom desempenho, sendo que todos os indivíduos que se encontram dentro dos limites são avaliados como tendo um desempenho normal ou desejável.

Ao colocar a ênfase na avaliação de determinada situação, diabetes, hipertensão, numa disciplina generalista como a nossa, é natural que os cuidados se foquem nestas áreas mesmo se a qualidade dos cuidados noutras áreas também exclusivamente clínicas como a asma, a osteoartrose, a cardiopatia isquémica, alterações funcionais do estômago, cistite precisem também ser melhoradas. Por outro lado, existe o problema de a população, incluída nos estudos que suportam a evidência dos indicadores escolhidos, não corresponder à população real com multimorbilidade e a tomar vários fármacos em simultâneo. Estes estudos excluem os sintomas dos doentes, o seu estado funcional, a severidade das suas doenças, as suas ideias e preferências, apesar destes factores afectarem as decisões dos médicos quanto ao tratamento mais apropriado. ${ }^{16,18,19}$

Talvez sejam algumas destas limitações que vários problemas têm criado para que a reforma dos CSP se possa implementar de forma mais célere. A discussão através da usfamiliar@yahoogrupos.com.br revela receios por parte de diversos profissionais. Uma das apreensões manifestadas relaciona-se com os denominadores de vários indicadores. Por exemplo, deverá haver avaliação de um determinado indicador, tendo por denominador toda a população inscrita na USF/UCSP, 
ou apenas os indivíduos vigiados nas mesmas? Como se poderá homogeneizar cada indicador, tendo por base populações diferentes, com problemas sociais diferentes? Poderá e deverá haver critérios e indicadores diferentes para cada unidade de cuidados ou para as USF e UCSP?

\section{INDICADORES DE DESEMPENHO PARA AS UNIDADES DE SAÚDE FAMILIARES. AS DÚVIDAS QUE SUBSISTEM}

O movimento a nível mundial para o alcance da melhoria da qualidade teve reflexos em diferentes sectores de actividade, levando cada empresa, governo e país a interrogar-se sobre o seu processo produtivo e adoptando estratégias de racionalização, visando a melhoria do seu desempenho face a um mercado mais competitivo. $\mathrm{O}$ «benchmarking» permite a comparação interna $\mathrm{e}$ externa dos resultados obtidos por diversos indicadores. Por isso, é útil que cada USF ou UCSP, em qualquer momento, possa obter dados de avaliação interna e em simultâneo os possa comparar com os obtidos por outras USF ou UCSP. Mas é necessário que esta comparação possa ser feita entre unidades com o mesmo perfil (exemplo, mesmo número de profissionais, mesmo número de utentes, mesma área geográfica) e com a possibilidade de se efectuarem filtros à amostra em comparação e à avaliação de cada parâmetro.

As agências de contratualização entenderão que se está ainda numa fase inicial de implementação da reforma dos CSP e que a experiência e a contribuição das USF em actividade são imprescindíveis para se detectarem erros, omissões ou imprecisões, propondo novas funcionalidades ou a retirada de alguns indicadores que não adicionem mais-valia ao processo e sugerindo processos mais eficientes de determinação de indicadores. Os resultados servem para alterar para melhor o que está a ser feito. As USF que não estão a atingir os objectivos devem ser ajudadas sendo que as agências de contratualização deveriam adoptar uma postura de «ensinar a pescar», mais medicina baseada na evidência e menos baseada em indicadores. Um doente que vem a uma consulta para vigilância de HTA pode usufruir de aconselhamento para a saúde e de outros cuidados antecipatórios? A mãe jovem que ontem acompanhou o seu jovem filho que apresentava uma tinha do couro cabeludo em tratamento, pode ser alvo de

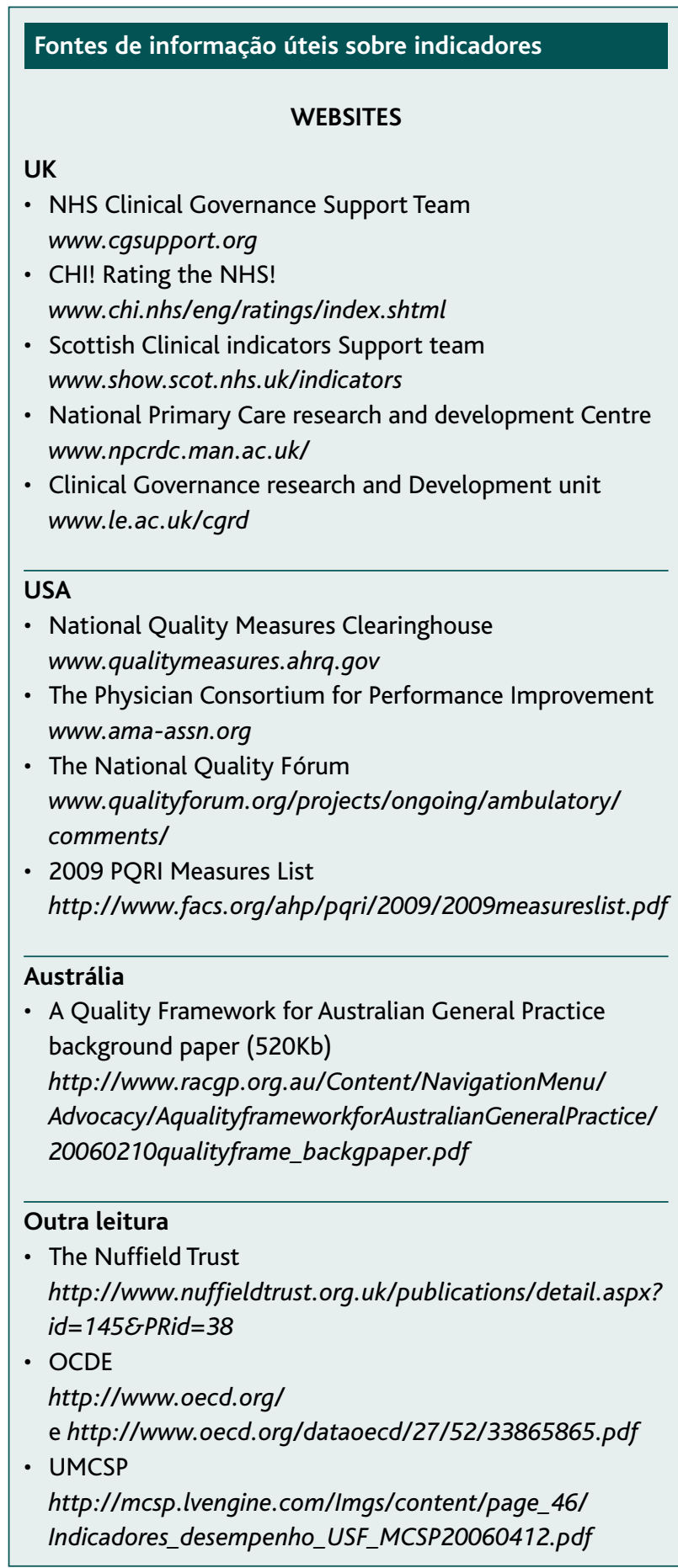

preocupação por parte do clínico quanto aos seus métodos contraceptivos actuais ou deverá ser marcada uma consulta de planeamento familiar porque há que 


\section{Mensagens-chave}

- É necessário avaliar a fim de melhorar a qualidade

- Alguns aspectos da MGF são mais facilmente mensuráveis que outros

- Existem problemas com o uso de indicadores de desempenho em CSP que precisam ser respondidos

- O uso de indicadores de desempenho e de qualidade poderão ser armas poderosas se forem usadas para a melhoria dos cuidados e não como processos de avaliação penalizadora

- Os indicadores não deverão ser vistos como a única ferramenta possível para se atingir a qualidade

cumprir tempos de consulta e não se pode fazer este registo na modalidade de planeamento familiar?

A percentagem de utentes activos difere muito de região para região e depende mais das características da população local do que do desempenho dos médicos. O quadro de avaliação desenhado encoraja os médicos e toda a equipa a concentrarem-se nos níveis de «scores» atingidos e nos benefícios económicos gerados para o grupo pelo cumprimento desses objectivos e baseia-se no pressuposto, irrealista, de que todas pessoas desejam ter uma esperança de vida o mais longa possível, quando na realidade alguns desejam morrer prematuramente e outros desejam adoptar comportamentos de risco, com plena consciência de que com isso estão a encurtar as suas vidas, colocando o futuro imediato à frente das incertezas dos ganhos a longo prazo. ${ }^{10}$

Apesar de alguns indicadores definirem limites de idades, pode-se ainda assim perceber que os níveis dos indicadores hemoglobina glicada e lipoproteina de baixa densidade encorajam ao sobretratamento das pessoas idosas justamente porque não consideram o estado psicológico e o contexto social, a gravidade ou o prognóstico, havendo assim perigo de causar maior stress e até induzir síncopes, quedas e fracturas. Uma revisão sistemática de literatura efectuada em 2000 sobre o efeito dos incentivos financeiros ${ }^{20,21}$ alerta para o aumento das desigualdades entre doentes e recomenda que se ajustem os incentivos financeiros à qualidade, produtividade e severidade dos doentes tratados, admitindo serem estes ajustamentos difíceis.
As unidades ponderadas, utentes inscritos na USF, justificam-se pela necessidade de maior equidade na sua distribuição, diz a $\mathrm{MCSP}^{2}$ e por isso o número de crianças até aos 6 anos é multiplicado pelo factor 1,5, as pessoas entre os $65 \mathrm{e}$ os 74 anos multiplicadas pelo factor 2 , as pessoas com mais de 75 anos multiplicadas pelo factor 2,5 e os restantes inscritos mantêm o factor 1 . Apesar de não se conhecerem os estudos epidemiológicos que fundamentaram as ponderações atribuídas, sabe-se que a idade e o sexo se relacionam com maior consumo de cuidados e de problemas crónicos de saúde. Mas estes não são os únicos determinantes de risco e por isso não deveriam ser as únicas variáveis para as quais se deveria fazer o ajustamento. A comorbilidade, a gravidade e a situação socio-económica também deveriam ser tidas em conta..$^{15,18,19}$ Multiplicarem-se os doentes com HTA pelo factor 2 e os doentes com diabetes mellitus pelo factor 4 , atendendo ao número de consultas que deverão ter num ano, fará esquecer outras patologias também gravemente incapacitantes e progressivas como por exemplo os doentes com doença pulmonar crónica obstrutiva ou com insuficiência cardíaca.

No cálculo das diferentes taxas, nos diversos indicadores, tão importante é definir o numerador como o denominador. Relativamente ao solicitado pelos Indicadores de Desempenho para as Unidades de Saúde Familiar publicado pela MCSP em 2006, deverá entrar-se com todos os utentes inscritos num dado médico ou apenas com os activos? Um utente acamado ou com uma doença terminal deverá receber a vacina antitetânica? Qual o critério para a obrigatoriedade de consultas trimestrais em doentes com Diabetes Mellitus se estes estiverem bem controlados? Será adequada a visita domiciliária de enfermagem ao RN e respectiva mãe puérpera, se tiver uma observação médica na primeira semana de vida? Que confiança os utilizadores poderão ter quando existem resultados diferentes nas taxas de vacinação consoante se utiliza o sistema SINUS ou SAM como fonte de informação?

Quando se usam dados e indicadores para avaliar o desempenho em MGF devem-se colocar diferentes perguntas, como refere Thomson. ${ }^{21}$ Do conjunto de perguntas efectuadas, a mais relevante será: os dados obtidos devem-se ao desempenho da unidade dos cuidados ou aos seus profissionais? O conjunto de indicadores 
parte de um princípio de bom senso, de aproveitar oportunidades e recursos. Mas será que podemos assumir que todas as intervenções sensatas conduzem necessariamente a ganhos em saúde? Temos disso provas?

\section{O QUE QUEREM OS DOENTES E O QUE QUEREM OS MÉDICOS DE FAMÍLIA?}

Quando um cidadão consulta o médico espera que este lhe providencie os melhores cuidados. As expectativas do doente face às possibilidades do médico ou da medicina dependem das suas crenças, da sua cultura, do ambiente social e económico em que vive, das políticas, das estratégias e da organização dos cuidados de saúde. O desempenho do médico depende do seu saber, da finalidade que atribui à sua missão, da interpretação que faz das necessidades da pessoa que o consulta e da organização dos serviços de saúde que presta.

A evidência mostra que os doentes como utilizadores de saúde têm prioridades distintas das que têm enquanto cidadãos pagadores de impostos. ${ }^{22}$ Em geral os cidadãos preocupam-se mais com a qualidade das interacções com os profissionais de saúde do que com a organização dos serviços. Por isso, é importante verificar em que medida a reforma prevista para os cuidados de saúde primários e a política de qualidade que se lhe segue pode potenciar ou minar os princípios do SNS e da MGF e aumentar os custos em saúde sem que isso se reporte em mais ou melhor saúde.

Uma revisão sistemática da literatura sobre as prioridades dos doentes em MGF, que examinou 19 estudos publicados entre 1966 e 1995, verificou que os factores mais valorizados pelos doentes eram, por ordem decrescente, o humanismo/humanidade, a competência/precisão, o envolvimento dos doentes nas decisões e o tempo de consulta com eles dispensado. ${ }^{23}$ Os doentes querem profissionais que comuniquem bem e que tenham conhecimentos e aptidões clínicas consistentes. Também desejam médicos que se interessem pelos seus problemas, os envolvam nas decisões, lhes dêem suficiente atenção e os aconselhem sobre auto-cuidado e promoção da sua saúde. Existem no entanto diferenças consideráveis na hierarquia das prioridades dos doentes face à Medicina Geral e Familiar. ${ }^{24}$ Os portugueses também realçam nas primeiras duas prioridades o tempo para ouvir, falar e explicar e a rapidez na resposta em casos de emergência, mas as suas restan-
QUADRO I. As dez prioridades dos doentes portugueses relativamente à Medicina Geral e Familiar $(n=3.540) *$

1. Durante a consulta o MF deve ter suficiente para me ouvir, falar comigo e me explicar

2. O MF deve ser capaz de prestar cuidados imediatos em caso de emergência

3. O MF não deve apenas curar doenças, deve também prestar serviços com vista a preveni-las

4. O MF deve frequentar regularmente cursos para se manter a par dos desenvolvimentos médicos

5. O MF deve ensinar-me a tomar os medicamentos

6. O MF deve fazer sentir-me à vontade para lhe dizer a ele ou a ela os meus problemas

7. O MF deve ter um interesse pessoal em mim e pela minha situação de vida

8. O MF deve garantir confidencialidade da informação acerca do seu/seus pacientes

9. O MF deve querer avaliar a minha saúde regularmente

10. Deve ser possível marcar uma consulta com o MF num curto espaço de tempo

*Ferreira PL, Raposo V. A governação em saúde e a utilização dos indicadores de satisfação. Rev Port Clin Geral 2006 Maio-Jun; 22 (3): 285-96.

tes oito prioridades já não obedecem à mesma hierarquia dos restantes países (Dinamarca, Alemanha, Israel, Holanda, Noruega, Reino Unido, Suécia e Portugal). Os doentes portugueses naquele estudo consideram como imediatamente prioritário, a vertente preventiva, a actualização dos médicos, dispensando--lhes tempo para se actualizarem e o ensinamento sobre a toma dos fármacos ${ }^{25}$ (Quadro I). Neste particular é importante lembrar que a avaliação do programa de cuidados farmacêuticos da $\mathrm{ANF}^{26}$ direccionado à diabetes, durante os primeiros 6 meses de 2006, contabilizou 212 comunicações de farmacêuticos a médicos por problemas relacionados com a medicação, tendo desta comunicação resultado 147 inícios ou alterações farmacológicas.

Portanto, é necessário ter em conta que a agenda do doente não corresponde à agenda do médico. Para o doente é importante que o seu problema, doença, sintoma ou queixa sejam resolvidos, para o médico pode ser mais premente naquela consulta a realização de actividades preventivas. A crença numa medicina tecnológica ajudada pelo marketing social, frequentemen- 
te apoiado por médicos, seguradoras, pela medicina desportiva, levam os cidadãos a pedirem exames auxiliares de diagnóstico com fraquíssimo poder prognóstico, pouco acessíveis, caros, sendo alguns até agressivos. Os médicos querem dispor de meios que lhes possibilitem efectuar diagnósticos atempadamente (o ecodoppler arterial e venoso não é comparticipado), querem dispor de um sistema de referenciação eficiente e de uma educação para a saúde que leve os cidadãos a compreender como lidar com os problemas de saúde minor mais frequentes. ${ }^{27}$ Como pode o médico de família satisfazer parte das necessidades dos doentes, como por exemplo cirurgias oftalmológicas ou ortopédicas, se não há resposta para estes pedidos nos cuidados de saúde secundários? Os médicos querem que a saúde não seja da sua única e exclusiva responsabilidade e que cada elemento e instituição social perceba o seu papel como determinante na qualidade da saúde e nos indicadores de desempenho dos médicos, sendo por isso que a saúde deve estar em todas as politicas.

\section{CONCLUSÕES}

A prática da avaliação de serviços, especialmente por meio de produção de estatísticas, mesmo que reconhecida e abraçada como actividade importante para o planeamento e gestão coordenada dos serviços de saúde, carece ainda de uma visão de futuro, orgânica e integradora, adequada às competências. Em tempos de recursos financeiros escassos, a eficiência e a eficácia são conceitos-chave para produzir evidência para os decisores, sendo por isso a padronização de medidas de desempenho a estratégia adoptada na procura de harmonia entre as métricas actuais de cada unidade de saúde. O presente trabalho mostra os erros que podem surgir de uma avaliação métrica que não tem em conta as particularidades da especificidade da MGF em particular a sua orientação para o contexto, a sua prática relacional, o seu potencial para se dirigir a problemas mal definidos e a sua capacidade para usar em simultâneo vários modelos de abordagem de saúde/doença consoante as circunstâncias particulares de cada um. Uma avaliação virada para indicadores de quantidade com incentivos financeiros de produtividade e ligados à prestação de determinados actos médicos pode conduzir a cuidados mais caros sem ganhos nos resultados clinicamente relevantes.

\section{REFERÊNCIAS BIBLIOGRÁFICAS}

1. Conceição C, Gonçalves A, Blaise P, Van Lerberghe W, Ferrinho P. A gestão do desempenho dos médicos de família no serviço nacional de saúde. Revista Portuguesa de Saúde Pública 2001 Jan-Jun; 19 (1): 15-23.

2. Ministério da Saúde. Missão para os Cuidados de Saúde Primários. Indicadores de desempenho para as unidades de saúde familiares. Lisboa: Ministério da Saúde; 2006. Dispoonível em: http://www.mcsp.minsaude.pt/NR/rdonlyres/EB139DE8-00B1-4B54-A19F-418D98D52DF0/ 3769/IndicadoresUSFMCSP2006412.pdf. [acedido em 30/04/2009].

3. Shön D. El professional reflexivo: como piensam los profesionales cuando actúan. Barcelona: Paidós; 1998.

4. Smith RC, Lyles JS, Gardiner JC, Sirbu C, Hodges A, Collins C, et al. Primary care clinicians treat patients with medically unexplained symptoms: a randomized controlled trial. J Gen Intern Med 2006 Jul; 21 (7): 671-7.

5. Sousa JC. Ups! Será que é MUPS? Rev Port Clin Geral 2006 Maio-Jun; 22 (3): 277-9.

6. Nunes JM. Carta ao Director. Rev Port Clin Geral 2006 Jul-Ago; 22(4):513-5.

7. Strumberg JP. The foundations of primary care: daring to be differernt. Oxford: Radcliffe Books; 2008.

8. Barry CA, Bradley CP, Britten N, Stevenson FA, Barber N. Patients' unvoiced agendas in general practice counsultations: qualitative study. BMJ 2000 May 6; 320 (7244): 1246-70.

9. Little P, Everitt H, Williamson I, Warner G, Moore M, Gould C, et al. Observational study Preferences of patients for patient centred approach to consultation in primary care: observational study. BMJ 2001 Feb 24; 322 (7284): 468-72.

10. Heath I, Hippisley-Cox J, Smeeth L. Measuring performance and missing the point. BMJ 2007 Nov 24; 335 (7629): 1075-6.

11. Baker M, Wilson T, Marshall M. The Royal College of General Practitioners. Policy Statement. Quality indicators in General Practice. Manchester: National Primary Care Research and Development Centre, University of Manchester; 2002.

12. Institute of Medicine, Crossing the quality chasm: a new health system for the 21st century. Washington, DC: National Academy Press; 2001.

13. Marshall M, Campbell C, Hacker J, Rolland M. Quality indicators for general practice. London: Royal Society of Medicine; 2002.

14. Pineault R, Daveluy C. La planification sanitaria: conceptos, métodos, estrategias. Barcelona: Masson; 1987.

15. Santos I. O doente com patologia múltipla em Medicina Geral e Familiar: comorbilidade de quatro doenças crónicas. Porto: BIAL; 2006.

16. Santos I. O desafio da comorbilidade para os serviços de saúde. Rev Port Clin Geral 2006 Mar-Abe; 22 (2): 191-4.

17. Cefalu WT,Watson K. Intensive glycemic control and cardiovascular disease observations from the ACCORD study: now what can a clinician possibly think? Diabetes 2008 May; 57 (5): 1163-5.

18. Starfield B. Co-morbidity and its challenge for quality of primary care Rev Port Clin Geral 2007 Mar-Abr; 23 (2): 179-80.

19. Gervas J, Santos I. A complexidade da comorbilidade. Rev Port Clin Geral 2007 Mar-Abr; 23 (2):181-9.

20. Caix-Couturier C, Durand-Zaleski I, Jolly D, Durieux P. Effects of financial incentives on medical practice: results from a systematic review of the literature and methodological issues. Int J Qual Health Care 2000 Apr; 12 (2): 133-42. 
21. Thomson R. Appropriate use of data: the example of indicators. In:Van Zwanenberg T, Harrison J, editors. Clinical Governance in Primary Care. 2nd ed. Oxford: Radcliffe Medical Press; 2004. p. 101-15.

22. Coulter $A$. What do patients and the public want from primary care? BMJ 2005 Nov 19; 331 (7526): 1199-201.

23. Jung HP, Baerveldt C, Olesen F, Grol R, Wensing M. Patient characteristics as predictors of primary health care preferences: a systematic literature analysis. Health Expect 2003 Jun; 6 (2): 160-81.

24. Grol R, Wensing M, Mainz J, Ferreira P, Hearnshaw H, Hjortdahl $P$, et al. Patients' priorities with respect to general practice care: an international comparison. European task force on patient evaluation of general practice (EUROPEP) Fam Pract 1999; 16 (1): 4-11.

25. Ferreira Pl, Raposo V. A governação em saúde e a utilização dos indicadores de satisfação. Rev Port Clin Geral 2006 Maio-Jun; 22(3):285-96.

26. ANF. Contributos em saúde para os diabéticos - avaliação do progra- ma de cuidados farmacêuticos: diabetes. Farmácia Observatorio, 2006 Ago. Disponível em: URL: http://www.anf.pt/images/stories/farmacia_observatorio/foespago06.pdf [acedido em 30/04/2009].

27. Dassow P. Measuring performance in primary care: what patient outcome indicators do physicians value? J Am Board Fam Med 2007 JanFeb; 20 (1): 1-8.

\author{
ENDEREÇO PARA CORRESPONDÊNCIA \\ Isabel Santos \\ Departamento de Clínica Geral \\ Faculdade de Ciências Médicas \\ Campo dos Mártires da Pátria, 130 \\ 1169-059 Lisboa. \\ Telf.: +351218 803061 \\ E-mail: isabel.santos@fcm.unl.pt
}

\begin{abstract}
Modern societies aim to gauge, quantify and evaluate the delivery of health care services they usually deal with.

Performance indicators measure components of the daily clinical work, which is central to establish priorities and management decisions and also to gauge the changes in health care systems.

Nevertheless the core issues are neglected, namely the doctor-patient relationship, the difference between what the patients perceives as the main problem and the issue that the doctor feels persuaded to approach, communication and the social context, among others.

The authors want do demonstrate what are the existent conceptual and practical problems behind family practice appraisals, should core characteristics of family medicine such as comprehensiveness, personalization, coordination and integration of care not be taken into account.
\end{abstract}

Keywords: Quality Indicators; Performance Indicators; Family Practice; Family Health Unit. 